\section{Electron Microscopy Enters a New Era Using Aberration Correction}

\author{
P.E. Batson \\ IBM Research, Yorktown Heights \\ batson@us.ibm.com
}

Once in a while we have the privilege of participating in an activity that promises to radically improve our technical capability to explore materials at the microscopic level. The recent practical demonstration of aberration correction in the SEM [1], the TEM [2] and the STEM [3] signify nothing less than a revolution in the way that we design and operate electron optics for microscopy.

Scherzer understood from the beginning of electron microscopy that an open magnetic lens having cylindrical symmetry will have positive, non-zero aberration coefficients, and so therefore will always have some minimum aberrations. [4] In practical instruments, it was arranged that the limiting defect would be spherical aberration in the objective lens. This allowed the imaging performance to be largely characterized by a single parameter, spherical aberration, and also allowed a certain amount of image simulation and post-experiment analysis to facilitate interpretation of imaging results. It led directly to the common practice of deliberately obtaining images out of focus (Scherzer defocus) to enhance transfer of finer crystal lattice detail - with the concurrent loss of longer ranged information about non-periodic features.

It was also understood that this situation could be fixed by giving up some of the symmetry elements of the cylindrical lens. One could use multi-pole lenses, put charge on the axis, use a mirror symmetry, or time-varying fields. Since Scherzer's work, there have been many attempts to implement these techniques with little practical success due to the extremely high precision needed to characterize and match the aberration characteristics of the microscope lenses. Finally, in the 1990 's, commencing with the work of Haider, we see the impact of several new capabilities becoming available: 1) electron optical calculation tools to handle complex optical systems, 2) mechanical fabrication techniques that allow more complex and reproducible pole-piece geometries, 3) cheaper, more reliable, and stable electronics for driving the new lens configurations, and 4) fast, local computation and control capabilities to allow nearly real-time characterization and control of aberrations during operation of the instrument. Taken together, these capabilities promise a revolution in the design, manufacture, and use of electron optical systems.

I describe here results using the Nion aberration corrector in the STEM where we achieved a remarkable electron probe smaller than $1 \AA$ for the first time. [5] This performance, about $0.8 \AA$, is 20 times the electron wavelength at $120 \mathrm{KeV}$ energy, breaking a barrier of about $50 x$ that had been imposed by $C_{S}$ in low voltage instruments. Atomic column imaging of semiconductor interfaces should be much easier now, using an acceleration voltage that is below the knock-on threshold for atomic displacement in Si.

The STEM is a good vehicle for investigation of corrector technology for several reasons. 1) The prime imaging mechanism, Annular Dark Field (ADF) imaging, relies primarily on elastic interaction with the positive charge at the atomic nucleus, and exhibits a non-oscillatory Contrast Transfer Function (CTF). Thus, there exists a simple relationship between the image resolution and the size of the electron beam at best focus. 2) Since the probe is formed
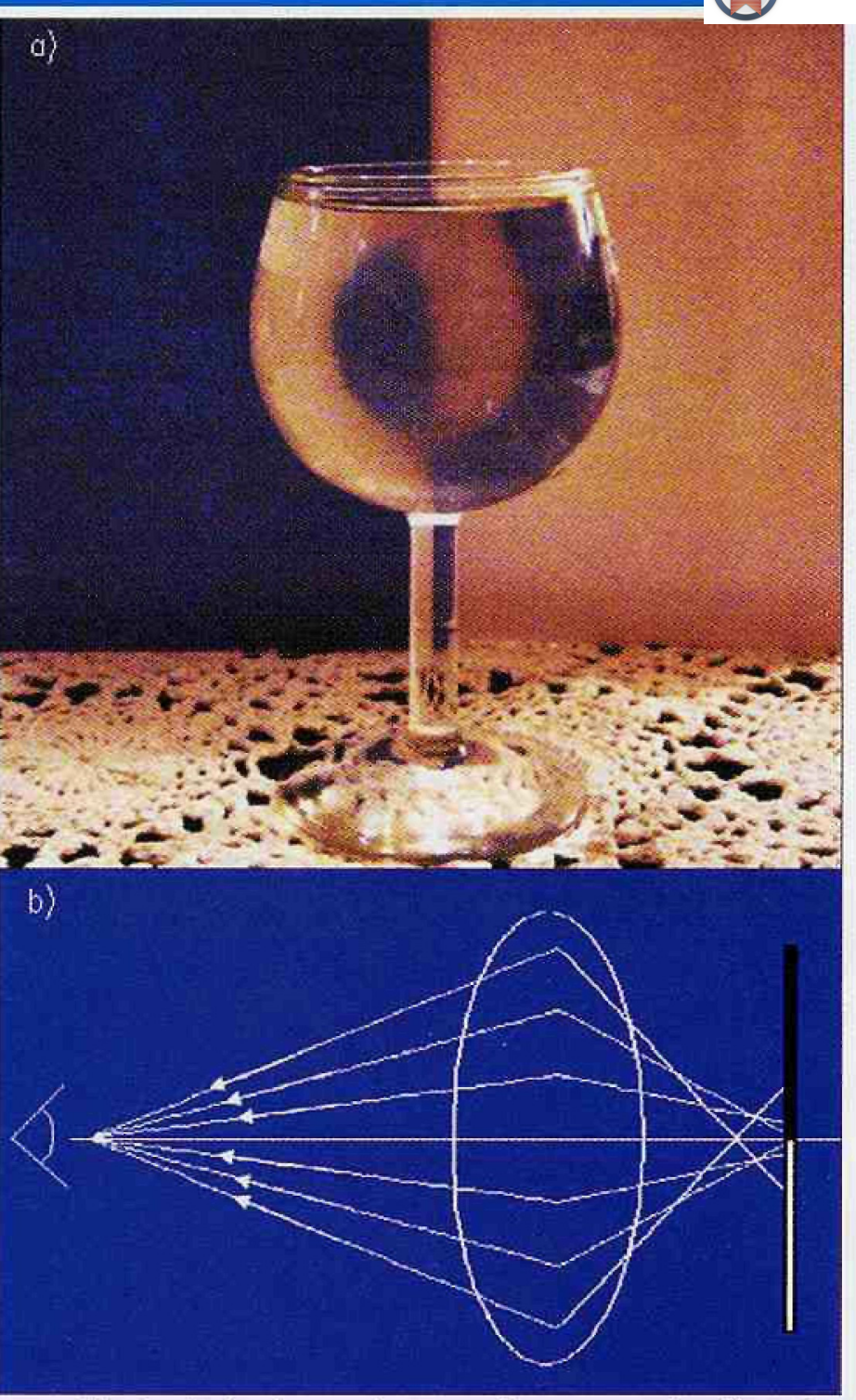

Fig. Ia. A simple experiment to demonstrate the effect of spherical aberration. The black-white card contrast is faithfully reproduced at small angles, where the focal plane of the lens is behind the card. At large angles, where the focal plane is in front of the card, the white-black regions are reversed. b) A schematic ray path representation to explain the above behavior.

before interaction with the specimen, aberration correction needs to be applied only close to the optic axis of the instrument, simplifying the complexity of the needed optics. 3) Also, because on-axis correction is sufficient, chromatic aberration is not a problem for sub-Angstrom resolution, eliminating the need for an electron energy monochromator to investigate the high resolution limits of the new system.

The IBM instrument is a VG Microscopes HB501UX STEM, delivered in 1981. This instrument has been modified in the past in the following ways: 1) a high resolution Wien filter spectrometer has been added; 2) the acceleration voltage has been increased to $120 \mathrm{kV}$ to improve specimen penetration and the gun optics; 3) the objective lens strength has been increased about $10 \%$ to improve the post-specimen compression for better spectrometer operation; 4) the $L N_{2}$ trapped diffusion pump has been replaced with a large ion pump; and 6) some simple computer control has been added to allow semi-automatic control of EELS acquisition to improve 


\section{TEMs Join the 4pi Revolution}

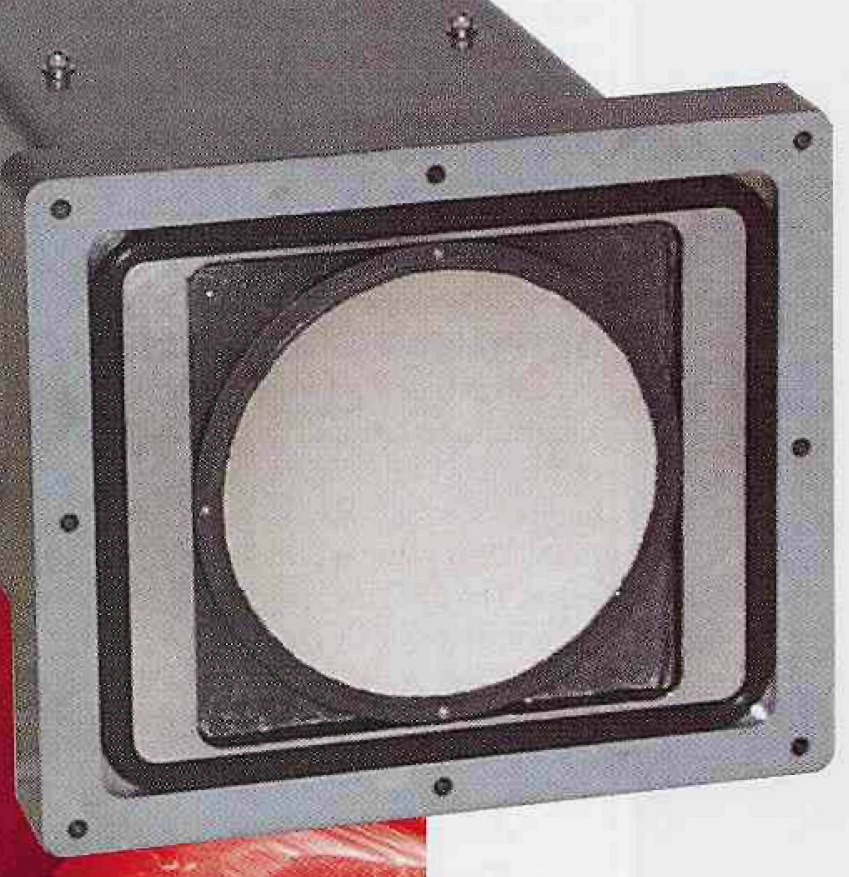

New CCD camera system

enables microscopists to acquire publication-quality digital images with an ease-of-use comparable to exposing film. Add affordability, flexibility (1.5 to 16 Megapixels), and choice of either Windows ${ }^{\oplus}$ or Macintosh ${ }^{\oplus}$ OS for a highperformance system unique to the marketplace.
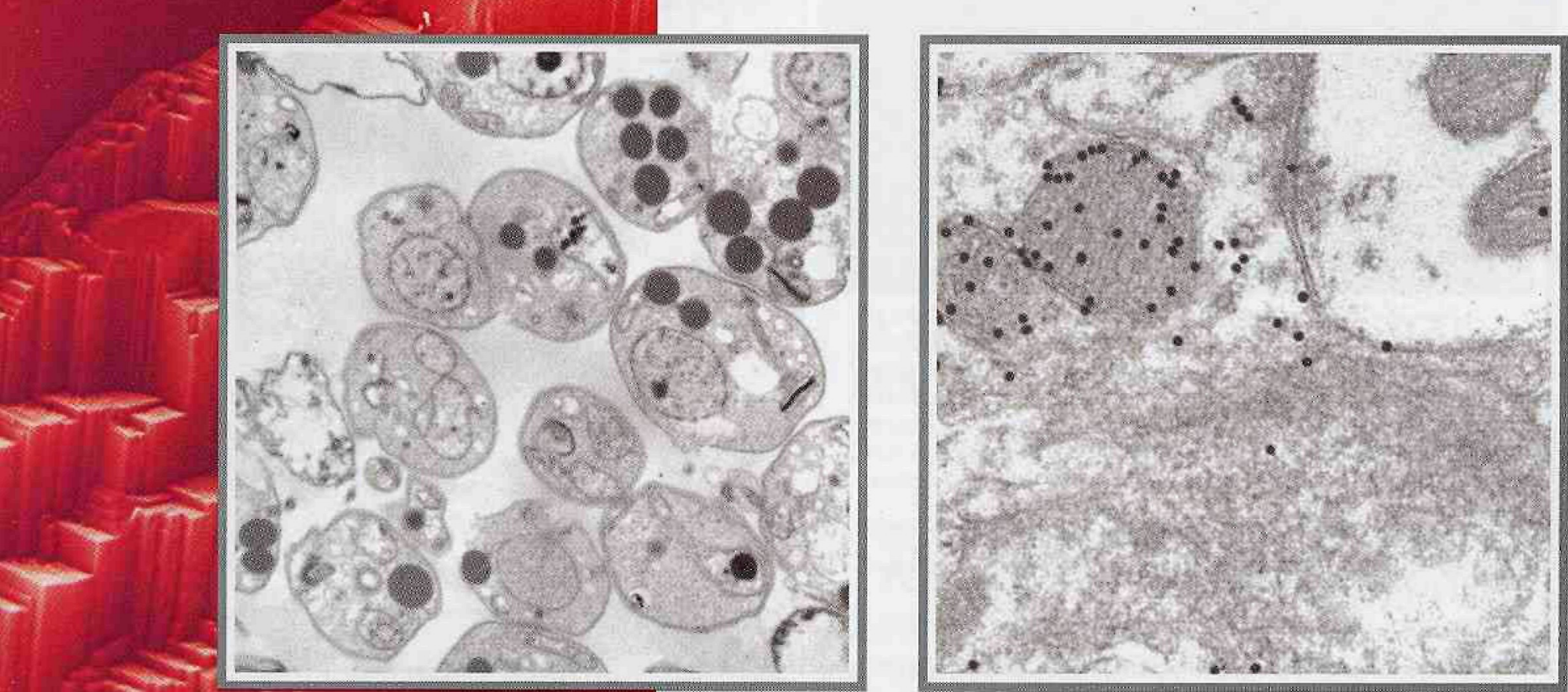

\section{EDX AND DIGITAL IMAGING}

High-resolution Systems and Upgrades for TEMs, SEMs, ESEMs and EMPAs. Innovation in desktop EDX and Imaging solutions since 1990 .

4pi Analysis, Inc.

3500 Westgate Dr. - Suite 403 - Durham, NC 27707 info@4pi.com • 919.489.1757 • www.4pi.com 


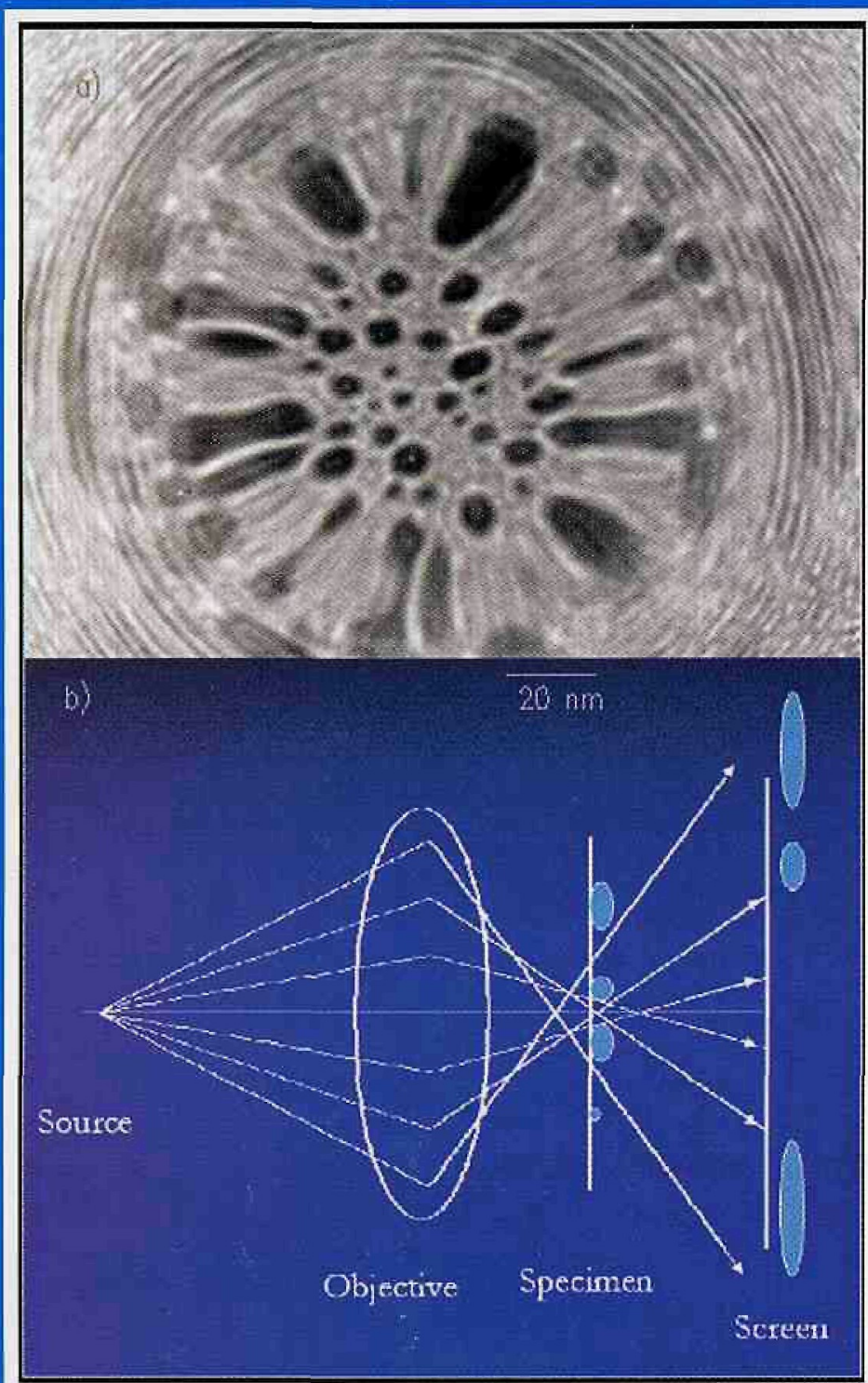

Fig. 2a. The electron "Ronchigram" is very similar to the aberrated image obtained in the wine glass experiment. Again, we see a region of simple imaging in the center of the figure, where, in this case, the beam crossover is behind the resolution test specimen (under-focus). A region having reversed contrast is apparent at large angles, where the beam is over-focused. Between the two regions is a region of infinite magnification.

reproducibility. The higher acceleration voltage and objective excitation delivered 1.9-2.0 $\AA$ resolution using $\mathrm{ADF}$ imaging. ( $C_{S}=$ $1.2 \mathrm{~mm}$ at $120 \mathrm{kV}$ ).

Fig. 1 summarizes a simple optical example to illustrate spheri$\mathrm{cal}$ aberration. The wine glass serves as a simple lens to image a black/white background. As is evident in Fig. 1b, rays that pass near the center of the lens reproduce the object faithfully, but rays that pass far from the center exhibit reversed white-black contrast. Between the two extremes, a ring of infinite magnification exists. The image shown in Fig. 1a, therefore, represents a map of both the object information and the lens aberrations.

A similar situation exists for an electron shadow map, the "Ronchigram". [6] In Fig. 2, I show the shadow map of a $10 \mathrm{~nm}$ gold island resolution test specimen using a probe focused behind the specimen. We see again that for small angles, a faithful image of the specimen is obtained. But for larger angles, the lens aberrations distort the sample features producing a reversal of magnification with a ring of infinite magnification at intermediate angles. If one obtains patterns similar to those of Fig. 2 a with sufficient accuracy under several conditions of focus or beam position, it becomes possible in principle to separate sample features from lens distortions, so that aberrations in the optical system may be fully characterized. In practice, these measurements cannot be made in enough detail to fully characterize the uncorrected system, but are instead used in an iterative fashion with a partially corrected system to indicate ever finer adjustments as the system moves closer to being fully corrected.

As shown in Figure 3, the Nion corrector is a quadrupole octupole system consisting of seven elements: four quadrupoles used to control the beam trajectory, and three octupoles used to adjust spherical aberration. The quadrupoles are adjusted to produce two orthogonal beams having pencil shapes, one in each of the two end octupoles, to allow the independent control of spherical aberration in the $(x, y)$ plane. At the midplane of the corrector a round beam exists. The third octupole is placed here to correct four-fold distortions introduced by the other two octupoles. Finally, a round beam is produced at the exit of the corrector. The corrector occupies a $15 \mathrm{~cm}$ long space originally used by the scan coils, which have been moved to within the bore of the objective lens.

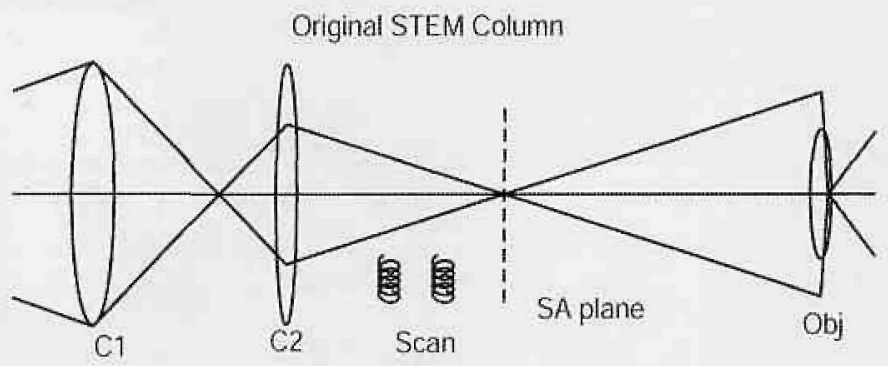

3rd Order Strength

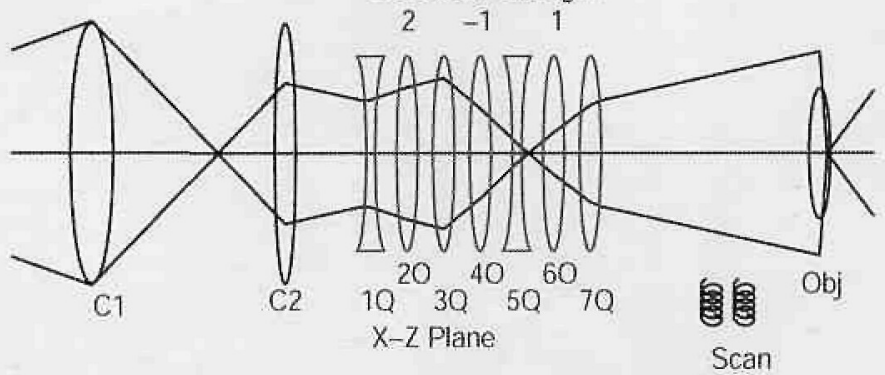

3rd Order Strength

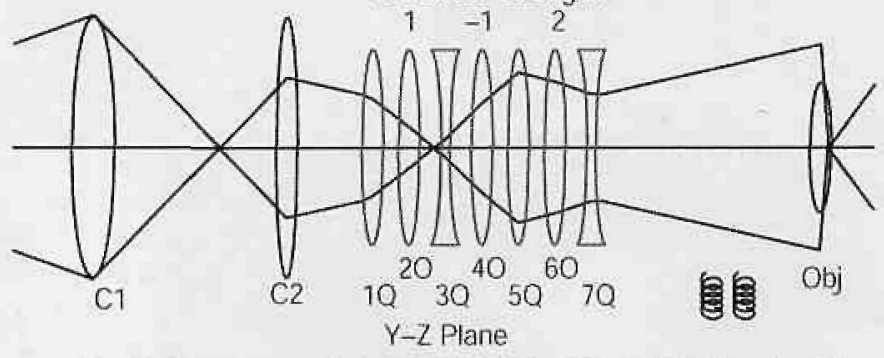

Fig. 3. Summary of the optics of the VG STEM fitted with a simple quadrupole-octupole corrector. The VG scan coils and selected area aperture have been replaced with the $15 \mathrm{~cm}$ lang set of 4 quadrupoles and 3 octupoles. The quadrupoles have been designed to produce cigar shaped beam cross sections in the first $(x$-direction) and third ( $y$-direction) octupoles, while preserving round beams at the center octupole and at the corrector exit. 
Although operation can be described using only the seven major lenses, non-uniformities and misalignments introduced in the construction of the corrector introduce parasitic aberrations that must be controlled using as many as 25 minor fields, bringing the total number of required windings to about 35 . With these extra windings, it is necessary to use software to drive several windings at once to produce controls that specify pure dipole, quadrupole and octupole fields of specified orientation and position relative to the optic axis. The windings are all excited separately using $0.3 \mathrm{ppm}$ stability, computer-controlled current supplies. The availability of this many controllable supplies in an affordable and manageable package is new to electron microscopy, and is largely a result of the explosion in capability that has become available in integrated electronics during the past ten years.

The capability to characterize the system in terms of many aberration parameters and ultimately to control as many of these as possible, demands a new descriptive nomenclature. In the uncorrected machine, we describe the shape of a probe wavefunction formed in the objective lens as

$$
\Psi_{p}(\vec{r}) \propto \int_{\text {apertire }} e^{-i \chi(\vec{k})-i \vec{k} \cdot \vec{r}} d \vec{k}
$$

where $\chi(\vec{k})$ is the phase shift for electrons traversing the lens as a function of the electron momentum $\vec{k}$ transferred to the sample. This vector has a magnitude that is roughly $K_{0} \theta$ with $K_{0}$ being the incident electron wavevector and $\theta$ being the scattering angle.

In the standard cylindrically symmetric description, where only spherical aberration, chromatic aberration and defocus are important, the direction of $\vec{k}$ is not important and we have

$$
\chi(\theta)=\frac{\pi}{\lambda} \theta^{2}\left[\frac{1}{2} C_{s} \theta^{2}-\left(\Delta f-C_{c} \frac{\Delta E}{E\left(1+E / m_{0} c^{2}\right)}\right)\right]
$$

where $C_{C}$ is the chromatic aberration coefficient, $\Delta E / E$ is the fractional energy spread of the beam and we have included a relativistic correction.

For $C_{S}=1.2 \mathrm{~mm}$, and electron wavelength, $\lambda=0.034 \AA$, we expect to achieve an $\mathrm{ADF}$ resolution:[7]

$$
d_{s} \approx 0.43 C_{s}^{1 / 4} \lambda^{3 / 4}=2.0 \AA \text { at } 120 \mathrm{KV} .
$$

For the aberration corrected machine, $C_{S}$ and $C_{c}$, are not general enough to characterize all the system aberrations. Therefore, Krivanek has suggested the following scheme: Let an aberration coefficient be numbered, $C_{n, m},(a, b)$ where $n$ denotes the radial exponent of the lens field strength, $m$ denotes the azimuthal symmetry and $(a, b)$ denotes the orientation of the azimuthal variation. Then, we can write a Taylor expansion for the phase shift,

$$
\begin{array}{r}
\chi(k, \phi)=K_{0} \sum_{n} \frac{\left(k / K_{0}\right)^{(n+1)}}{(n+1)} \sum_{m}\left[C_{n m a} \cos (m \phi)+C_{n m h} \sin (m \phi)\right] \\
\text { with }\left\{\begin{array}{c}
m+n \text { odd } \\
m<n+1
\end{array}\right.
\end{array}
$$

In this scheme the first order coefficients $C_{1}$ and $C_{12 a, b}$ define defocus and astigmatism; coma becomes $C_{21 a, b}$ and $C_{3}$ identifies spherical aberration.
In the optimally adjusted instrument, corrected for spherical aberration, the 5 th order aberration limits performance:[3]

$$
d_{5} \approx 0.43 C_{5}^{1 / 6} \lambda^{5 / 6}=0.6-0.9 \AA \text { at } 120 \mathrm{KV} \text {, }
$$

for $C_{5}$ of $1.6-12 \mathrm{~cm}$. The IBM machine gives measurements of $C_{5}$ on the low end of this range.

In the end, the microscope operation requires an approximate setup using previously known corrector settings to begin the day. Then, detailed measurements of Ronchigram shadow maps provide aberration coefficients that are used to refine the corrector settings, taking the instrument in steps to a fully focused condition. The iterative procedure helps to minimize in-
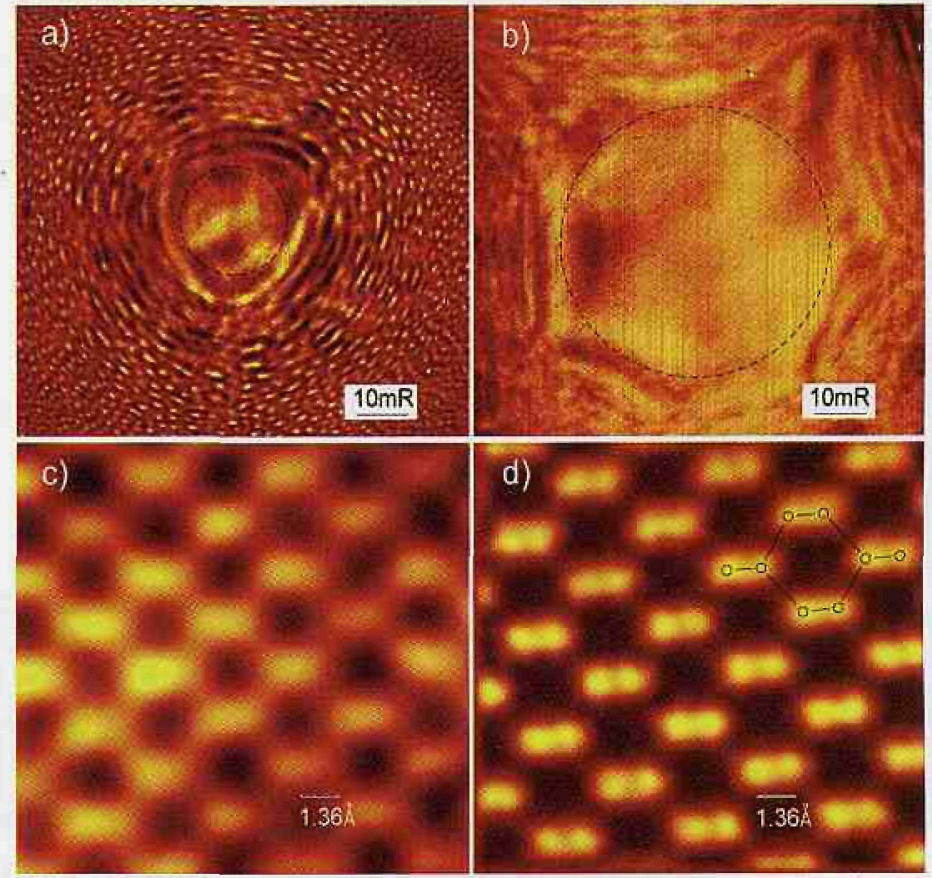

Fig. 4. Comparison between uncorrected and corrected results for the Ronchigram at focus $(a, b)$ and $\mathrm{Ge}_{30} \mathrm{Si}_{70}$ imaging. $(c, d)$ Averaging has been used in both images to reduce ac interference and to suppress acquisition noise. The corrected results show spatial frequencies out to about $0.8 \AA$.

stabilities and inaccurate calibrations (for instance Ronchigram camera length, lens field strengths, gun demagnification). Fig. 4 shows a comparison of imaging results for a $\mathrm{Ge}_{30} \mathrm{Si} 70$ alloy. In the upper two panels, Ronchigram results at focus are shown. The region of low contrast in the center of the pattern indicates a range of angles where the phase of the transmitted electrons is constant. In the uncorrected instrument, this extended out to about $10 \mathrm{mR}$, consistent with about a $1.9-2.0 \AA$ resolution. In the corrected instrument, this area covers angles out to $25 \mathrm{mR}$, or about $0.7 \AA$, in agreement with the rough prediction of $\mathrm{Eq} .5$ above.

Fig. 5 shows a more typical result for a $\mathrm{Si} / \mathrm{Ge}_{30} \mathrm{Si}_{70}$ quantum well interface. This is more difficult to obtain since we are interested in a particular area of the sample. A wider dynamic range for the image data is necessary, as well, because intensity variations are important in the image for understanding possible atomic number contrast. In contrast with the $2 \AA$ resolution re- 
data. [8] Thus, for these very high resolution experiments, we need to rethink data acquisition, probe scanning strategies, data display and storage. To this end, the IBM system now uses a relatively fast $1024 \times 800,10$ bit acquisition system, running at 1-10 fps. Image data and acquisition parameters are stored using a tiff structure that has been designed to be accessible via most imaging software.

What has become abundantly clear is that aberration correction produces spectacular improvements in our capability. In response, the way in which the instruments are used has changed dramatically towards more precise and reproducible operation. Finally, the additional detail requires us to rethink how we acquire, display, analyze and store the resulting image data.

I wish to acknowledge extensive collaboration with O.L. Krivanek and N. Dellby who are responsible for this corrector design and construction, and collaboration with $\mathrm{Z}$. $\mathrm{Hu}$ and J. Silcox towards a better understanding of the effects of non-linearity in the data acquisition.

[1] J. Zach and M. Haider, Optik, 112 (1995).

Fig. 5. Ge ${ }_{30}$ Sizo/Si quantum well interface illustrating a practical level of performance at this time. The $1.35 \AA$ atomic pair distance is resolved, and the atomic number contrast is strong. Statistical variations are more prominent than in the $2 \AA$ results, and there is a greater sensitivity to nonlinearity in signal acquisition.

[2] M. Haider, S. Uhlemann, E. Schwan, H. Rose, B. Kabius, \& K. Urban, Nature, 768 (1998).

[3] N.Dellby, O.L. Krivanek, P.D. Nellist, P.E. Batson, and A.R. Lupini, J. Electron Microscopy , 177 (2001).

[4] O. Scherzer, J. Appl. Phys., 20 (1949).

[5] P.E. Batson, Niklas Dellby, and O.L. Krivanek, Nature, 617 (2002).

[6] V. Ronchi, Applied Optics, 437 (1964).

[7] J. Silcox, P. Xu, and R.F. Loane, Ultramicroscopy, 173 (1992).

We saw in these early results that non-linearities in obtaining this data can produce non-physical spatial frequencies in the

[8] Z. Yu, P.E, Batson, and J. Silcox, Ultramicroscopy, 275 (2003).

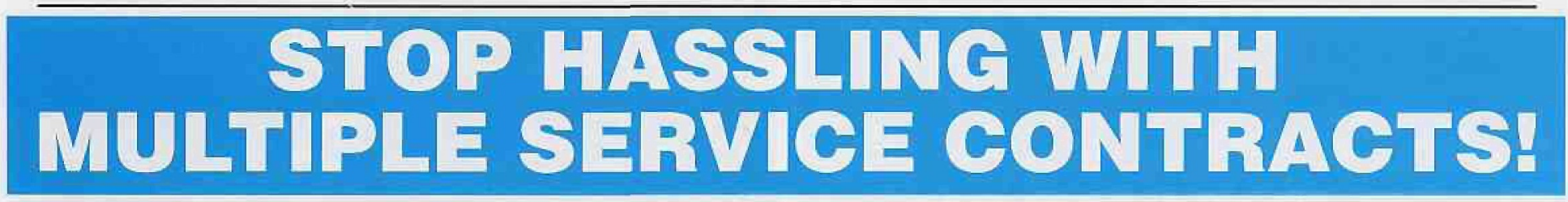

START by putting all of your instruments under one service contract with MAS (regardless of make or model). Our expert EM SERVICE GROUP has the knowledge and skills to keep your instrument working at its best.

\begin{tabular}{|cc|c|c|}
\hline \multicolumn{2}{|c|}{ TEMIS / SEMIS } & PREP EQUIPMENT & SPEOIAL SERVICES \\
\hline HITACHI & TOPCON & VACUUM COATERS & STAGES \\
JEOL & ISI & PLASMA ASHERS & BEAM BLANKERS \\
AMRAY CAMBRIDGE & SPUTTER COATERS & CUSTOM DEVICES \\
\hline
\end{tabular}

\section{NEW NEW NEW}

\section{Authorized service representatives for Gatan preparation equipment and Topcon TEM'S.}

Contracts and On-Demand Emergency Service at Reasonable Rates from Factory Trained Specialists.

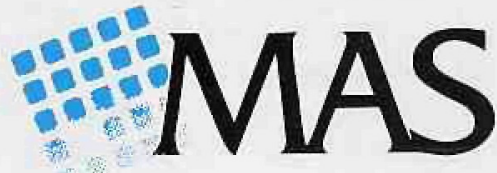

\section{0-421-8451}

3945 Lakefield Court Suwanee, Georgla 30024 770-866-3200 FAX 770-866-3259 616 Hutton Street Suite 101 Ralcigh, North Carolina 27606 919-829-704I FAX 919-829-5518 ADVANCED ANALYTICAL PRODUCTS AND SERVICES 


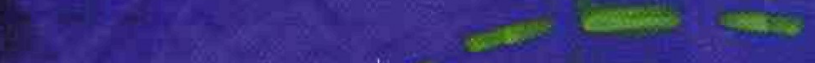

\section{THE NEXT GENERATION}

I MAG ING F I LTER

HAS ARRIVED

\section{PRECISION}

Gatan proudly announces GIF Tridiem, our Third Generation of post-column energy filters. GIF Tridiem combines $3^{\text {rd }}$-order spectrometer aberration correction with a multi-port, high-speed, high-resolution CCD sensor to yield a system that defines a new state-of-theart in the capture of highly-detailed EELS and EFTEM data sets with maximum throughput. GIF Tridiem, combined with the Gatan Microscopy Suite (GMS) software, is ideally suited for generating and working with rich 3-dimensional data sets, including EFTEM and EELS STEM spectrum images, EFTEM tilt and tomography series, and time series. GIF Tridiem makes tri-dimensional EM readily accessible to the most demanding analytical electron microscopist.
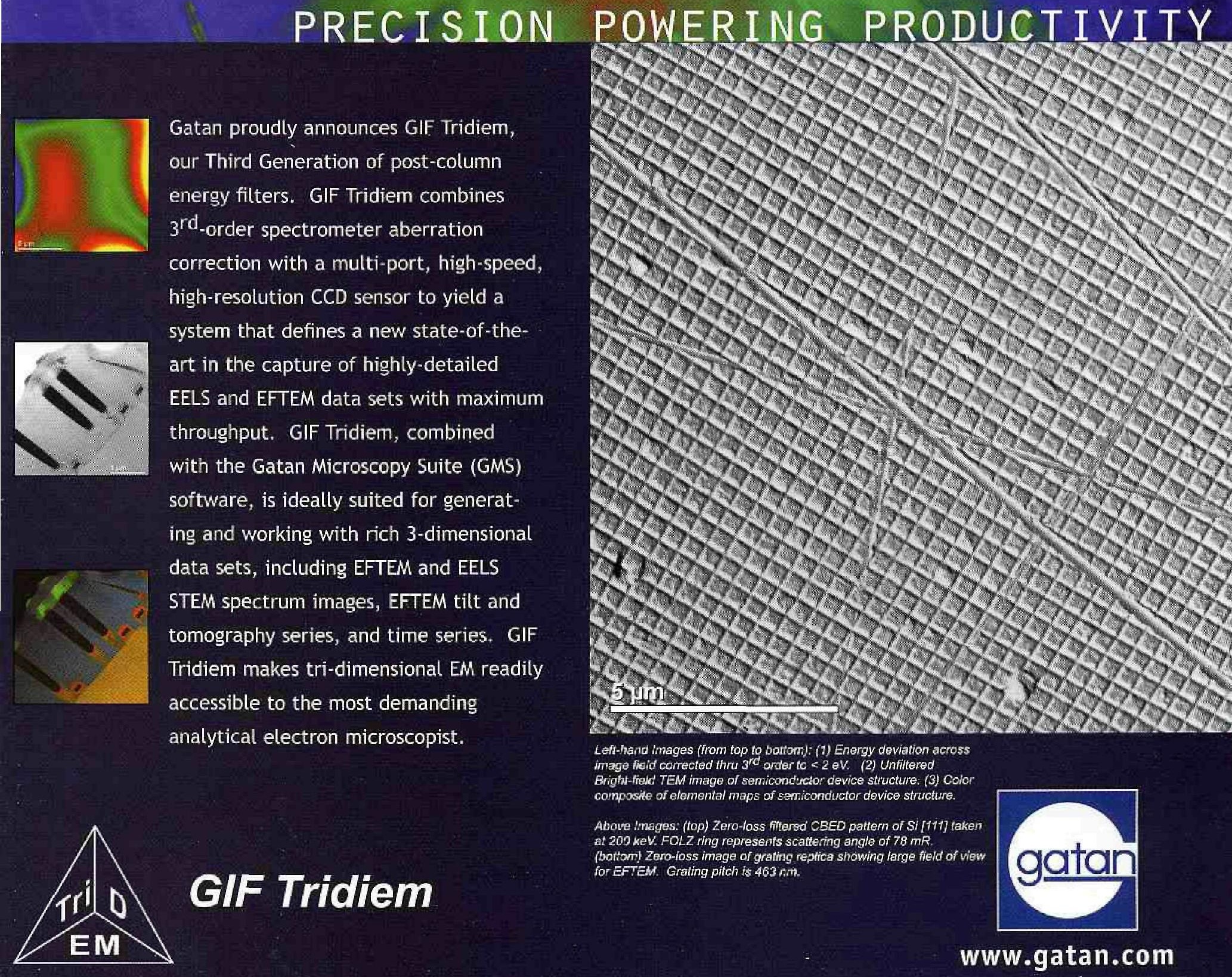

Leth-hand images (rom top to bottom): (1) Energy deviation across image fiald conrected thru $3^{\text {rd }}$ order to $<2 \mathrm{eV}$. (2) Unfiltered Bright-field TEM image of semiconductor device structure: (3) Color composite of elemental maps of semiconductor device structure.

Above Images: (top) Zero-loss fitered CBED pattern of Si [117] laken at $200 \mathrm{keV}$. FOLZ ring represents scattering angle of $78 \mathrm{mR}$. (bottom) Zero-loss image of grating replica showing large field of view for EFTEM. Graling pich is $463 \mathrm{~nm}$.

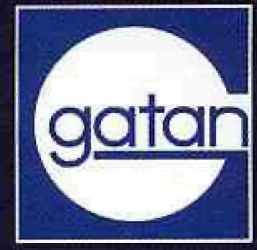

